\title{
The Potential for Image Guided Radiation Therapy with Cobalt-60 Tomotherapy
}

\author{
L. John Schreiner ${ }^{1,2}$, Andrew Kerr ${ }^{1,2}$, Greg Salomons ${ }^{1}$, Christine Dyck, and \\ George Hajdok ${ }^{3}$
}

1 Medical Physics Department, Kingston Regional Cancer Centre

25 King St W., Kingston, ON, Canada, K7L 5P9

2 Departments of Physics and Oncology, Queen's University

3 Department of Medical Biophysics, University of Western Ontario 790 Commissioners Rd. E., London, ON, Canada, N6A 4L6

\begin{abstract}
Helical tomotherapy, a new approach for Intensity Modulated Radiation Therapy, employs a fan-beam of radiation from a source mounted in a CT-like ring gantry. Complex conformal dose delivery is achieved by modulating the intensity of the radiation beam as the source revolves about the patient. A particular benefit of helical tomotherapy is the ability to perform in-situ CT imaging to confirm patient set-up, and to reconstruct the dynamically delivered dose distributions. In this paper we present the results of ongoing work to establish the potential for tomotherapy using a Cobalt-60 radioactive source. Both dose delivery and megavoltage CT imaging data confirm the feasibility of image guided radiation therapy using Cobalt-60 tomotherapy.
\end{abstract}

\section{Introduction}

There are about 4000 new cases of cancer in North America per year per one million population. A major treatment for cancer is radiation therapy, and approximately $50 \%$ of all cancer patients receive radiation at some time during their illness. In radio-therapy, tumours are treated by radiation from an x-ray unit or radioactive source. The goal of treatment is to deliver a sufficient and uniform dose to the target (to achieve tumour control) while minimizing the dose to normal tissue (to avoid complications). In the last four decades, radiation therapy practice was improved: i) by the move from low energy radiation beams to more penetrating megavoltage beams, ii) by the development of improved imaging to localize tumours, and iii) by improved radiation dosimetry. It has been proposed that external beam radiation therapy can be further improved by the precise selection of the number of radiation beams and their geometry so as to obtain a closer conformation of the delivered dose distribution to the target volume, that is, by the implementation of conformal radiation therapy techniques [1]. For a given photon beam energy, three main physical parameters may be manipulated to achieve a distribution which better conforms to the desired volume: i) the number and orientation of the radiation beams, ii) the shape of each beam, and iii) the intensity of the radiation within each beam. A special implementation 
of conformal therapy, which manipulates all three of these parameters, is called Intensity Modulated Radiation Therapy (IMRT). To date IMRT techniques have been developed almost exclusively with linear accelerator (linac) x-ray units.

\subsection{Tomotherapy and Cobalt Therapy}

Tomotherapy is an implementation of IMRT proposed by Mackie et al. [23] based on a rotational IMRT delivery using a fan beam of radiation. Radiation modulation is provided by a binary vane multi-leaf collimator (MLC) and, in helical tomotherapy, the radiation source is mounted in a CT-like gantry. Complex three dimensional dose distributions through a volume are achieved by modulating, under computer control, the radiation intensity across the fan beam as the source revolves around, and the patient is advanced through, the gantry.

The potential for 4 or $6 \mathrm{MV}$ linac based helical tomotherapy has been well established [3,4. An important feature of tomotherapy is that the treatment geometry provides a convenient means to perform on-line computed tomography (CT) for patient set-up verification and dose reconstruction. This is achieved by the addition of an arc of detectors in the gantry opposite the radiation source. Patient imaging is achieved by reconstructing exit beam data (after the patient) during a pre treatment scan using a constant low intensity irradiation. Dose reconstruction, i.e., imaging of the delivered dose distribution, is achieved by reconstruction of the exit beam data acquired during the IMRT delivery [3]. Research in megavoltage computed tomography (MVCT) with a linac based tomotherapy unit has shown that high fidelity CT images can be obtained with a reasonable scan time and dose 5 .

The ability for patient position verification and dose reconstruction with tomotherapy suggests that the technique is particularly well suited for adaptive radiation therapy. Unlike other treatment modalities, radiation therapy is given in multiple daily visits throughout the course of their care: a patient typically returns to the radiation unit from 16 up to 40 times during treatment. In adaptive radiation therapy [67, one uses imaging data acquired during the treatment session and, perhaps, dose delivery data to assess how well the planned treatment has been achieved and to provide correction (see outline in Fig. 1). A simple version of the approach is to image the patient immediately prior to dose delivery, compare the on-line image with the CT images used for treatment planning, and correct the patient position if agreement is not within some set tolerance. A more sophisticated approach would be to adjust the treatment plan (i.e., the 'program' driving the intensity modulation) to accommodate the new patient position. If one is able to determine the dose delivered during a given treatment, it may also be possible to adjust subsequent radiation delivery to correct for deviations of the dose delivery from the plan, for example, by adjusting the intensity modulation the next day to add additional dose to volumes that received doses lower than planned, or to give less radiation through volumes that were overdosed. In tomotherapy, the MVCT imaging provides all the information required for adaptive radiation therapy. Investigations have shown that even without image reconstruction the exit beam data acquired by the detection system (i.e., the 
acquired sinograms) provide sufficient information for assessment of the setup and dose delivery 38 .

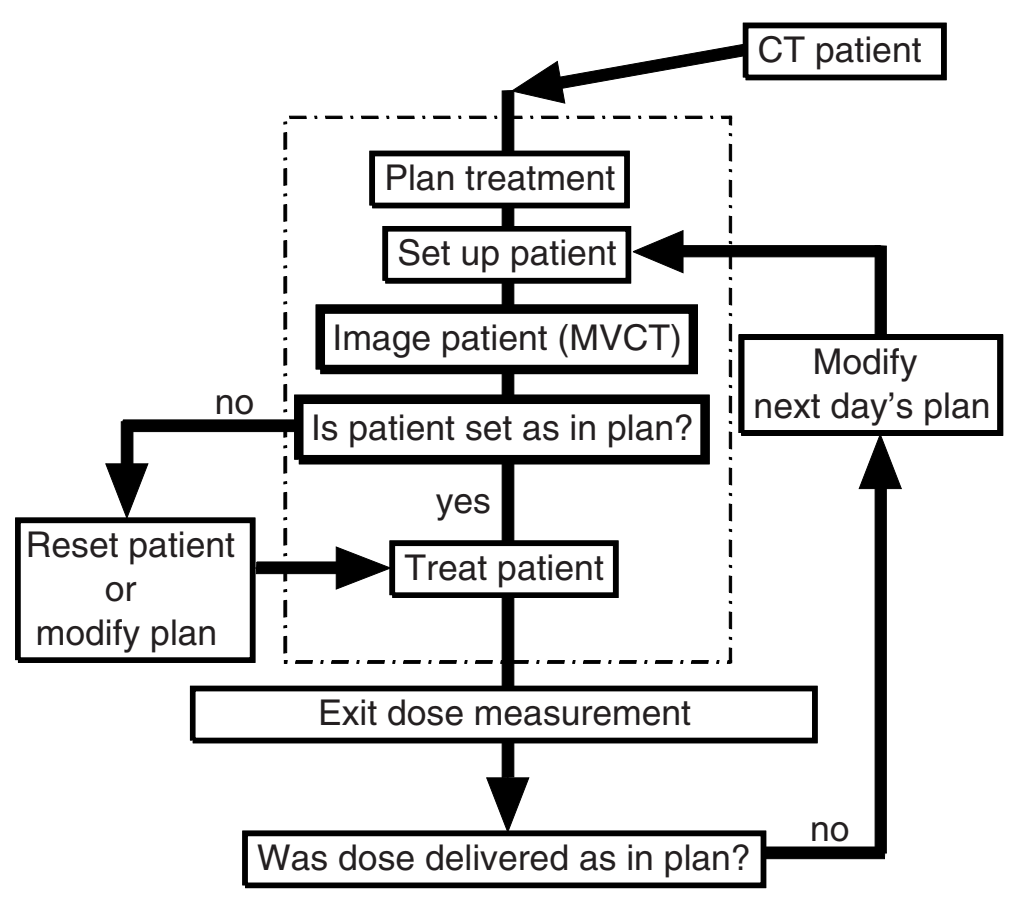

Fig. 1. The process of adaptive radiation therapy (see text) in tomotherapy. The two aspects indicated in the frames with heavy borders are inherent with the rotational geometry of tomotherapy. Image guidance at treatment is achieved through MVCT of the patient. If the patient setup agrees with the treatment planning then treatment can proceed directly. If not then the patient can be moved or the treatment plan can be modified. Our current investigations are devoted to assessing Co-60 for the features in the dashed box.

The use of radioactive Cobalt-60 (Co-60) in radiation therapy applications has a fifty-year long history. However, Co-60 has steadily fallen out of favour in clinical practice over the last two decades. This has not been because of the properties of the radiation beam, but rather because Co-60 units have not kept pace with modern progress in treatment technology 9], in particular, there has been little investigation of the potential for Co-60 conformal therapy. The medical physics group at the Kingston Regional Cancer Centre has aimed to modernize Co-60 radiation therapy by investigating the viability of Co-60 as the radiation source in a tomotherapy device [10[11].

In this work we present results of the investigations of the potential of tomotherapy using a Co-60 source for the dose delivery and megavoltage photon 
imaging required for adaptive radiation therapy. The goal is to develop a robust, readily available, technology that could extend the use of sophisticated adaptive techniques to smaller radiation clinics in Canada and the USA and to developing countries.

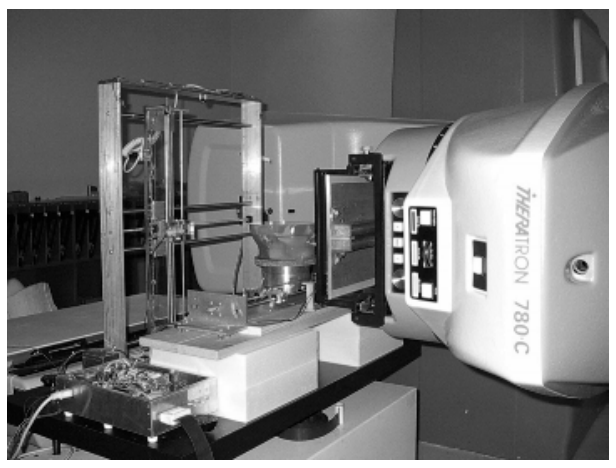

Fig. 2. The components that comprise the benchtop Co-60 tomotherapy system. This device mimics a tomotherapy treatment unit by rotating and translating the phantom in three dimensions through a $1 \times 1 \mathrm{~cm}^{2}$ pencil beam. The $2 \mathrm{D}$ scanning diode detector apparatus, on the left, can scan a single detector in a plane to perform fan (and cone) beam CT measurements.

\section{Materials and Methods}

Investigations were performed using computer modeling and simulation along with measurements on a first generation benchtop Co-60 tomotherapy device, shown in Fig. 2 The test device rotates and translates a phantom through a $1 \mathrm{~cm}^{2}$ pencil beam, or a $1 \times 35 \mathrm{~cm}^{2}$ fan beam, with $1 \mathrm{~mm}$ translational and $1^{\circ}$ rotational accuracy. Translating a phantom through the pencil beam with variable translation speed mimics the intensity modulated fan beam of helical tomotherapy. Image reconstruction and computer simulations of imaging and of dose delivery were generated using in-house software written in the MatLab programming language (Math Works, Natick, MA).

For CT imaging, an ISORAD n-type diode detector (Sun Nuclear Corp., Melbourne, FL) is added after the phantom to acquire transmission measurements. The detector is kept stationary for imaging with the pencil beam (in a $1^{\text {st }}$ generation CT geometry) or can be scanned in 1 dimension for fan beam $\left(2^{\text {nd }}\right.$ generation CT) imaging. The apparatus provides a simple, low-cost alternative to a commercial detector array and is sufficient for assessing the viability of Co-60 MVCT. Various types of phantoms (both in-house and commercial) have been imaged.

A P-type Si diode with a $2.5 \mathrm{~mm}$ diameter and a $0.3 \mathrm{~mm}^{3}$ volume (Scanditronix Wellhofer, Bartlett, TN) was used to measure beam data required for the 
treatment planning. Additional beam measurements were performed with calibrated Kodak XV film (Eastman Kodak, Rochester, New York) digitized using a Vidar film scanner (Vidar Systems Corp., Hinden, VA). A modified MilanBentley technique [13] was used as the dose calculation engine in the treatment planning software. The algorithm uses the semi-empirical depth dose data (well approximated by a zero field size depth dose for the Co-60 pencil beam) and cross beam profiles at multiple depths (modeled as a double Gaussian) to calculate delivered doses.

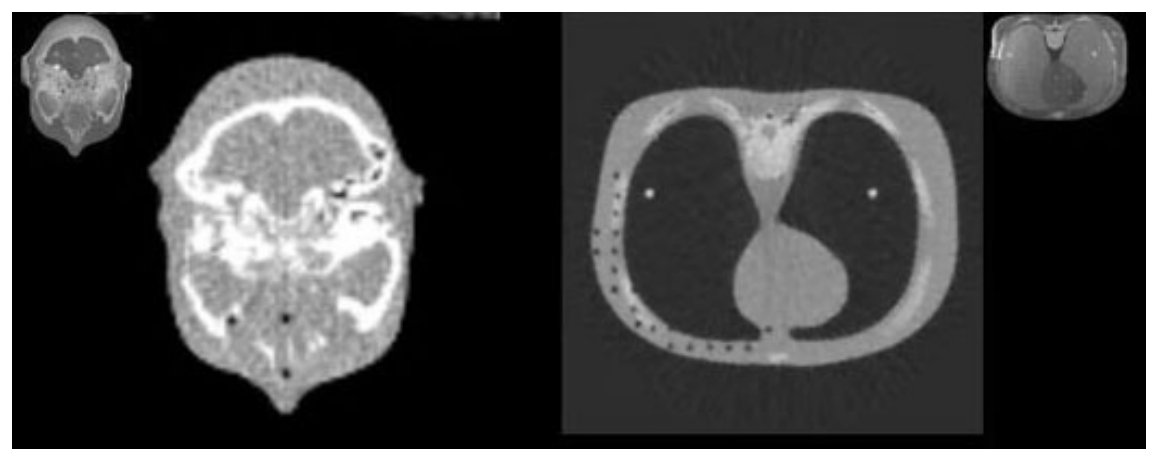

Fig. 3. Co-60 MVCT images from pencil beam imaging with the Co-60 benchtop unit. The images are through slices of an anthropomorphic phantom used for radiation dosimetry, the inserts show photographs of the corresponding slices. $3 \mathrm{~mm}$ air holes for TLD placement are clearly observed.

\section{Results and Discussion}

The intent of the current work is to show that Co-60 tomotherapy potentially provides the features required to undertake adaptive radiation oncology. To that end we need established that Co-60 provides: i) MVCT image quality sufficient to localize a patient during treatment, and ii) the ability to deliver conformal dose distributions. We indicate the MVCT results by showing typical images (in Figs. 3 and 4). Small high contrast features are readily seen in the images (i.e., the holes for thermoluminescent dosimeters in Fig. 3. and the glass containing methanol in the $20 \%$ contrast inserts in Fig. 41). These images illustrate that Co-60 MVCT provides images useful for the accurate patient position determinations required for adaptive therapy.

The accuracy of the treatment planning simulations of dose delivery have been validated against measurements, and shown to be accurate [13]. The median difference between in-house predicted and measured dose was $2 \%$ ( $<1$ cGy); in high gradient regions, the planned and delivered isodose lines are within a fraction of a millimeter. The ability to deliver complex conformal dose distributions 


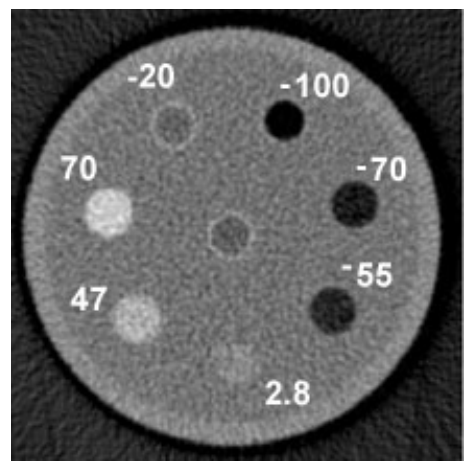

Fig. 4. A $2^{\text {nd }}$ generation fan beam MVCT image of a quality assurance phantom containing various $3 \mathrm{~cm}$ inserts. The numbers specify the differences of each material's electron density compared to water (e.g., bottom insert has $2.8 \%$ contrast).

is indicated by a simulation of a clinically relevant treatment of a head and neck tumour. The intent of the treatment is to deliver $100 \%$ of the dose to a mass on the right side of the neck while treating a node on the contralateral side to only $70 \%$. Figure 5 shows that the desired dose distribution can be achieved with the Co-60 tomotherapy unit. The dynamic IMRT treatment delivery required to achieve the dose distribution in Fig. 5 is illustrated in Fig. 6. In this figure the output of the in-house MatLab treatment planning software has been captured for four specific orientations as the tomotherapy beam revolves about the targets. The right hand image in each pair shows the intensity modulation achieved by the translation through the pencil beam at the given angle, i.e., this image illustrates the fan beam intensity modulation inherent to tomotherapy. The left hand image shows the integration of the dose as the source revolves about the patient. At $360^{\circ}$ the left hand image would give the dose distribution shown in Fig. 5. Although the grey scale specifying dose in each image varies with angle, the convention is that white shows high intensity or high dose regions in the right and left images in each pair, respectively. The dose delivered at each orientation is on the order of $2 \%$ of the total dose.

\section{Conclusions}

Our investigations of Cobalt-60 IMRT continue to confirm the strong potential for Co-60 tomotherapy. Acquired images, including those of anthropomorphic phantoms, confirm that Co-60 MVCT provides the anatomical visualization necessary for adaptive radiation therapy. The conformal dose delivery capabilities are also as required. Thus, a Co-60 tomotherapy device provides the major components required of adaptive radiation therapy delivery. Future research will be to examine dose verification from reconstruction of exit dose data, and to advance the benchtop unit by incorporating a multileaf collimation for fan beam IMRT and a detector arc for $3^{\text {rd }}$ generation MVCT. The next step will be to incorporate 



Fig. 5. A test of conformal dose delivery with the Co-60 tomotherapy unit. The physician has delineated two targets in a head and neck treatment, one to receive $100 \%$ of the dose while the other receives only $70 \%$. The left hand image shows isodose contours on the CT image of the slice being treated, the right hand side shows the same information in a gray scale wash.
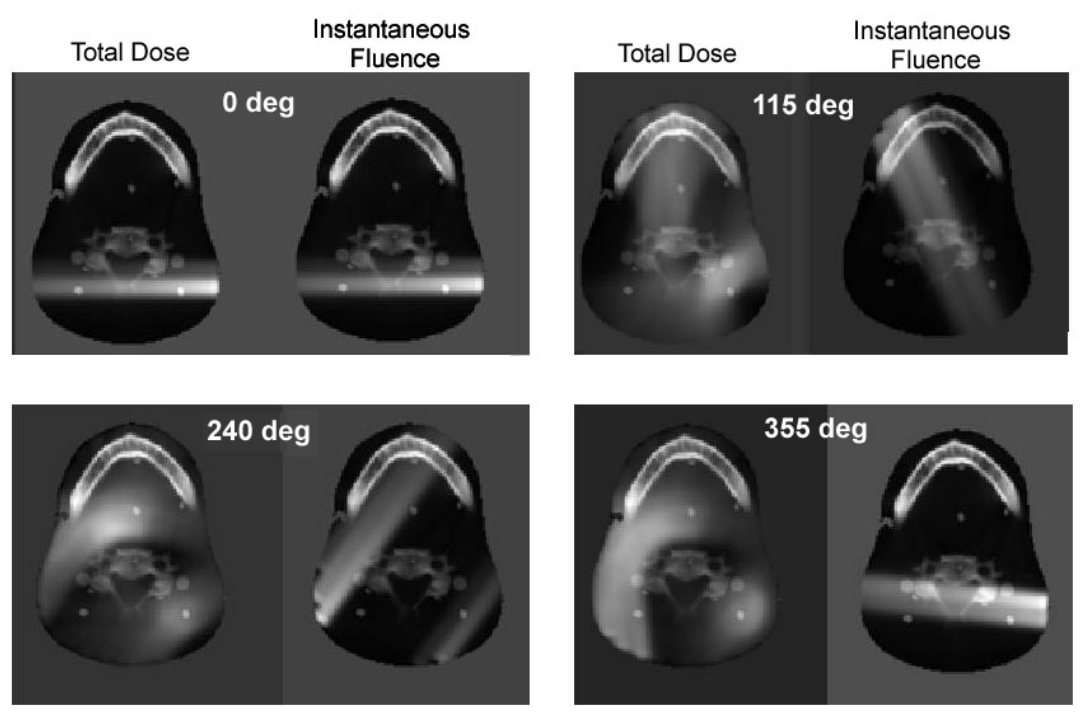

Fig. 6. Four frames from a movie generated by the treatment planning software illustrating the dynamic Co-60 tomotherapy dose delivery required to achieve the distribution in Fig. 5 In each pair the right hand image shows the instantaneous fan beam modulation pattern required at specific orientations as specified in degrees. The left hand image shows the total dose as the delivery progresses. The dose distribution of Fig. 5 is achieved after a complete rotation. The gray scale changes with each image to maintain dynamic range. 
the components from our enhanced benchtop unit into our clinical Co-60 unit to investigate the practical implementation of Co-60 tomotherapy. The feasibility of the required modifications to the Co-60 unit are being assessed.

Acknowledgements. This work is funded by the Canadian Institutes of Health Research, the Dering Cancer Research Fund, Theratronics/Cancer Care Ontario Research Initiative, the ORDCF's Ontario Consortium of Image Guided Therapy \& Surgery.

\section{References}

1. Webb S. The Physics of Three-Dimensional Radiation Therapy. Bristol, UK: IOP Publishing Ltd, 1993

2. Mackie, T.R., T. Holmes, S. Swerdloff, P. Reckwerdt, J. O. Deasy, J. Yang, B. Paliwal, and T. Kinsella, Tomotherapy: a New Concept for the Delivery of Dynamic Conformal Radiotherapy. Med. Phys. 20, 1709-1719, (1993);

3. Olivera G.H., D.M. Shepard, K. Ruchala, J.S. Aldridge, J. Kapotoes, E.E. Fritchard, P.J. Reckwerdt, G. Fang, J. Balog, J. Zachman and T.R. Mackie, Tomotherapy. In. Modern Technology of Radiation Oncology. J. Van Dyk (ed.) Madison WI: Medical Physics Publishing, 1999

4. Yang, J.N., T. R. Mackie, P. Reckwerdt, J. O. Deasy, and B. R. Thomadsen, An Investigation of Tomotherapy Beam Delivery. Med. Phys. 24, 425-436, (1997);

5. Ruchala, K.J., G. H. Olivera, E. A. Schloesser, and T. R. Mackie, Megavoltage CT on a Tomotherapy System. Phys. Med. Biol. 44, 2597-2621, (1999);

6. Lof J, Lind BK, Brahme A. An adaptive control algorithm for optimization of intensity modulated radiotherapy considering uncertainties in beam profiles, patient set-up and internal organ motion. Phys. Med. Biol. 43 1605-1628, (1998)

7. Martinez A.A., D.Yan, D. Lockman, D. Brabbins, K. Kota, M. Sharpe, D.A. Jaffray, F. Vicini, and J. Wong, Improvement in dose escalation using the process of adaptive radiotherapy combined with three-dimensional conformal or intensitymodulated beams for prostate cancer. Int. J. Radiat. Oncol. Biol. Phys. 50, 12261234; (2001)

8. Mackie T.R., J. Balog, K. Ruchala, D. Shepard, S. Aldridge, E. Fitchard, P. Reckwerdt, G. Olivera, T. McNutt and M. Mehta. Tomotherapy. Semin. Radiat. Oncol. 9, 108-117, (1999)

9. Van Dyk, J. and J. J. Battista, Co 60 : An Old Modality, a Renewed Challenge. Current Oncology 3, 8-17, (1996);

10. Kerr, A.T., G. J. Salomons, and L.J. Schreiner, Dose Delivery Accuracy of a Scanned Pencil Beam for Co 60 Tomotherapy Studies. In Proc. 47th Annual Conference of COMP, June 2001, 179-181, COMP, Edmonton AB;

11. Salomons, G.J., G. Gallant, A. Kerr, and L.J. Schreiner, CT Imaging for Co 60 Helical Tomotherapy. In CD Proc. World Congress on Medical Physics, July 2000;

12. Milan, J. and R.E. Bentley, The storage and manipulation of radiation dose data in a small digital computer, Brit. J. Radiol., 47, 115-121, (1974);

13. Salomons, G.J., A.T. Kerr, G. Hajdok, M. Rogers, C. Dyck and L.J. Schreiner, Further Progress in Cobalt-Tomotherapy At KRCC. In Proc. 49th Annual Conference of COMP, June 2003, in press, COMP, Edmonton AB; 